\title{
INDEX OF AUTHORS
}

Page numbers in italics refer to contributions, the others to the reference list at the end of papers

Aamodt, G. S., 332

Abramowitz, M., 356

Ahmad, M., 248

Akeley, R. V., 112

Alexopoulos, C. J., 103

Alfert, M., 372

Allan, L. D., 280

Allard, R. W., 71, 117, 118, 127, 331, 332

Allen, G. E., 33, 43

Allen, J. A., 401-404, 404

Allen, W. R., 348

Ames, B. N., 411

Angseesing, J. P. A., 73-83, 83

Anonymous, 112, 169

Antonorics, J., 296, 319, 348

Archer, L. J., (Ed.), 130-131

Arlett, C. F., 238

Arnold, M. H., 73, 74

Arnold, R. W., 169

Arrighi, E., 121

Ar-Rushdi, A. H., 269

Arst, H. N. Jr., 284, 285

Arthur, A. E., 396

Assouad, W., 44

Athwal, R. S., 181

Ayala, F. J., 108, 127

Ayonoadu, V. W., 121, 372

Babbel, G. R., 127

Bailey, C. R., 284

Bailey, N. T. J., 331, 332

Baker, B. S., 386

Baker, H. G., 33

Bancroft, J. B. (Ed.), 421-422

Bantock, C. R., 217

Barclay, I. R., 269

Bard, M., 283

Barlow, B. A., 331

Barnes, B. W., 127, 209

Bashaw, E. C., 332

Battaglia, E., 260

Beardmore, J. A., 156

Beckman, L., 401

Bendich, A., 373

Ben-Shaul, Y., 238

Berick, R., 238

Bernet, J., 103

Berry, R. J., 10

Bhanu, B. V., 145-149, 149

Bhattacharya, P. K., 372

Bird, L. S., 72

Birley, A. J., 122-127
Bishop, J. A., 83, 307

Blank, L. M., 72

Bliss, C. I., 93

Bloch, D. P., 373

Boncinelli, E., 143

Bonner, J., 373

Borgaonkar, D. S., 332

Bosemark, N. O., 260

Boyd, E., 280

Boyer, H. W., 269

Bradshaw, A. D., 296, 297, 309-319, 319, 348

Brat, S. Ved, 225-230

Breese, E. L., 296, 419

Brenner, M., 411

Brewbaker, J. L., 401

Brewer, G. J., 108

Brink, R. A., 143

Brinkerhoff, L. A., 72

Broker, W., 296, 348

Brooks, M. H., 332

Brown, A. H. D., 321-333, 332

Brown, M. F., 261

Brown, S. J., 53-72, 72

Brown, W. L., 406

Brumpton, R. J., 208

Bukhari, A. I., 412

Burton, G. W., 188

Burton, W. G., 112

Busch, H., 373

Cain, A. J., 169, 170, 217

Callan, H. G., 164, 386

Carlquist, C., 33

Carpenter, A. T. C., 386

Caspari, E. (Ed.), 273-274

Catcheside, D. G., 230, 386

Caten, C. E., 103, 223

Cauderon, A., 269

Cauderon, Y., 269

Ceppellini, R., 278

Chandley, A. C., 164

Chapman, V., 181

Charlesworth, B., 127

Charlesworth, D., 127

Chase, S. S., 414

Chigusa, S. I., 127

Church, Kathleen, 159-164, 164

Cionini, P. G., 373

Clarke, B., 404

Clarke, B. C., 170

Clark, J., 104

Clayton, G. A., 348 
Clegg, M. T., 122, 127

Cleland, R. E.,287-291

Cloaston, T. W., 72

Cochran, W. G., 83, 94, 307

Cockerham, C., 108

Cockerham, C. C., 157

Cocking, E. C., 104

Collins, G. B., 269

Collins, O. R., 104, 238

Connor, H. E., 33, 43

Cook, L. M., 307

Cook, S. C. A., 296

Cooper, J. E. K., 121

Corkill, L., 83

Cornish, E. A., 93

Correns, C., 33, 43

Cove, D. J., 284

Cox, B. S., 239

Grawford-Sidebotham, T. J., 83

Croft, J. H., 223

Growe, L. K., 51

Cummins, S. E., 104

Cunningham, C. E., 112

Currey, J. D., 169, 170

Daday, H., 83

Daniels, K. M., 249

Darlington, C. D., 121, 230, 271-272 (Bk. rev.), 272-273 (Bk. rev.), 273-274 (Bk. rev.)

Darwin, C., 10

David, T. J., 281

Davies, D. R., 267-270, 414

Davies, D. S. (Ed.), 421-422

Davies, R. W., 356

Davis, P. E., 10

Day, P. R., 104

Deasy, D., 112

Dee, J., 238

Dempsey, E., 387

Dessureaux, L., 296

De Wet, J. M. J., 332

Dickinson, A. G., 296

Diez, J. L., 373

Dilling, W., 348

Dobzhansky, Th., 307, 406

Dommée, B., 44

Drew, J. P., 112

Dronamraju, K. R., 43

Dulbecco, R., 238

Durrant, Alan, 133-143, 143, 276 (Bk. rev.)

Dutta, P. C., 149

Dyer, A. F., 386

East, E. M., 33

Eaves, L. J., 208

Eckhart, W., 238

Edwards, J. H., 277

Eglington, E. G., 143

Ehrman, L. (Ed.), 273-274

Eig, A., 181
Elandt-Johnson, R. G., 332

Elkington, T. T., 43

Ellis, J. R., 43

El-Zik, K. M., 72

Emara, Y. A., 219-224

England, F. J. W., 183-188

Evans, G. M., 143, 373

Evans, H. J., 260

Evans, L. T., 332

Falconer, D. S., 419

Ferguson-Smith, M. A., 280

Fincham, J. R. S., 104, 143

Finlay, K. W., 93

Fisher, R. A., 43, 208, 409

Fitzsimmons, D. W. (Ed.), 271-272

Fontenot, J. F., 112

Ford, C. E., 238

Ford, E. B., 217

Foster, C. A., 188

Foulds, W., 83, 281

Fowell, R. R., 248

Fox, D. P., 386

Franklin, I., 127

Freeman, G. H., 94, 208

Freire-Maia, N., 149

Frieden, C., 401

Friis, J., 248

Fripp, Yvonne, 94

Frolich, E., 348

Frydenberg, O., 108, 157

Fulmali, P. M., 145-149, 149

Fyfe, J. L., 332

Gall, J. G., 121

Gale, J. S., 208, 389-396, 396

Garnjobst, L., 104

Gartside, D. W., 287-297, 319, 335-348

Gehenio, P. M., 238

Gerlach, W. L., 241-249

Geschwind, I. I., 372

Giannelli, F., 279

Gibson, J. B., 356

Gibson, S. L. M., 281

Gilmore, R. S., 373

Giménez-Martín, G., 387

Godley, E. J., 33, 43

Goldin, B. R., 401

Goodman, R. N., 104

Graziani, F., 143

Gregory, R. P. G., 296, 319, 348

Grell, E. H., 127, 230

Grell, R. F., 164

Grewal, M. S., 33, 43

Grewal, N. S., 248

Griffing, J. B., 296

Grime, J. P., 83

Grindle, M., 238

Grun, P., 261

Gunatilleke, I. A. U. N., 285 
Gupta, S. B., 269

Gustafsson, A., 332

\section{Hadley, H. H., 72}

Haga, T., 261

Hagemann, R., 143

Hakim, S. M., 149

Haldane, J. B. S., 386

Hamilton, D. G., 270

Hannon, N., 348

Harding, J., 332

Harlan, J. R., 332

Harper, J. L., 44

Harris, H., 104, 127, 238

Harris, W., 43

Harrison, B. J., 83, 143

Hart, G. E., 401

Harvey, P. H., 401-404

Haslam, J. M., 283

Haupt, W., 164

Hayes, G., 281

Hayman, B. I., $72 \quad$ 179, 296

Hayward, M. D., 188, 296

Head, J. J. (Ed.), 129-130

Heath, D. J., 299-307, 307

Heddle, R. G., 200

Henderson, S. A., 164, 386

Henney, H., 238

Henney, M. R., 238

Heslwo-Harrison, J., 332, 386

Hewitt, G., 261

Hewitt, G. M., 230, 261

Hillel, J., 217

Himes, M., 373

Hirayoshi, I., 43

Ho, K. M., 270

Hold, G., 72

Holt, E. C., 332

Hopwood, D. A. (Ed.), 421-422

Hornby, C. A., 387

Horne, R. W. (Ed.), 421-422

Hovin, A. W., 332

Howell, J. I., 104

Hsu, T. C., 121

Huang, R. C., 373

Hubby, J. L., 127

Humphreys, M. O., 396

Hunter, R. E., 72

Ikeda, Y., 249

Inge-Vechtomov, S. G., 248

Ingle, J., 282

Innes, N. L., 53-72, 72

Innocenti, A. M., 373

Ishihara, S., 149

Jain, S. K., 33, 117

Jeffries, V., 282

Jenkins, S. J., 409-412
Jessop, A. P., 386

Jinks, J. L., 72, 143, 208, 209, 223, 224, 238, 296, 419

Joham, H. E., 72

Johansen, R. H., 112

John, B., 164, 230, 261

John, P. C. L., 373

Johnston, T. D., 275-276 (Bk. rev.)

Jones, D. A., 83, 104

Jones, G. H., 375-387, 386, 387, 422-423 (Bk. rev.)

Jones, J. S., 165-170, 170

Jones, R. N., 121, 129 (Bk. rev.), 357-373, 373

Jones, T. W. A., 143

Jones-Mortimer, M. C., 409-412

Jordan, C. A., 401-404

Jowett, D., 319

Kahan, B. D., 104

Kahlez, A. L., 118, 127

Kao, K. N., 270

Kaplan, S. M., 43

Kasha, K. J., 270

Kate, S. L., 145-149, 149

Kayano, H., 261

Kearsey, M. J., 127, 209, 419

Kendall, M. G., 94, 127, 332

Kermicle, J. L., 143

Kerr, N. S., 238

Khan, M. S. I., 296, 297, 309-319, 348

Khanna, K. R., 414

Kihara, H., 43, 181

Killick, R. J., 109-112

Kimber, G., 181, 414

Kimura, M., 108, 158

King, J. M. B., 217

Kirk, D., 373

Kirk, D. J., 357-373, 373

Klement, K., 104

Knight, R., 94

Knight, R. L., 72

Knox, R. B., 332

Kojima, K., 127, 157

Koltin, Y., 238

Korn, M. E., 83

Kovalenko, V. F., 373

Kusangi, A., 373

Lack, D., 10

Lakovaara, S., 108

Lamm, R., 373

Lange, W., 270

Lakovaara, S., 108

Lamm, R., 373

Lange, W., 270

Law, G. R. T., 401

Lawrence, C. W., 43

Lawrence, M. J., 209, 389-396, 396

Lederberg, J., 239

Lee, B., 282 
Lee, T. O., 224

Lefébvre, C., 296

Leng, E. R., 348

Lerner, I. M., 108, 157

Lewis, D., 33, 44, 51, 117

Lewis, K. R., 164, 261

Lewontin, R. C., 108, 127, 157

Lima de Faria, A., 121

Lindegren, C. C., 248, 249

Lindegren, G., 248, 249

Lindsley, D. L., 127, 230, 387

Ling, H., 95-104, 238

Ling, M., 95-104

Linnert, G., 33

Linney, R., 209

Lipke, W. G., 72

Lloyd, D. G., 11-34, 33, 35-44, 44, 45-51, 51

Lloyd, L., 164

Löve, A., 33, 44

Lowenstein, O. E. (Ed.), 129-130

Lucy, J. A., 104

Lundquist, A., 188

Lunt, O. R., 348

Lusnak, K., 249

Luyet, B. J., 238

Lyon, M. F., 143

McCarthy, J., 281

McCusker, A., 33

McDonald, P. T., 230

McKee, D. W., 249

McLeish, J., 373

McLellan, J. C., 143

McMillan, I., 349-356

McNeilly, T., 287-297, 296, 319, 335-348, 348

McWilliam, J. R., 188, 332

Magoon, M. L., 414

Malhotra, K. C., 145-149, 149

Malva, C., 143

Manly, B. F. J., 404

Manney, T. R., 248

Marchi, P., 122

Markham, R. (Ed.), 421-422

Marshall, C., 296

Marshall, D. R., 321-333, 332

Marshall, R., 280

Marushige, K., 373

Mather, K., 296, 332, 387, 406, 409, 414-419, 419

Mayr, E., 404-406, 409

Mettler, L. E., 127

Miksche, J. P., 373

Miller, J. C., 112

Miller, J. H., 412

Miller, J. J., 248

Miller, O. L., 387

Mitchel, J. P., 373

Moens, P. B., 387

Mohler, J. D., 44

Monesi, V., 373

Montgomery, R. D., 83
Moore, G. P. M., 373

Morris, J. A., 348

Morris J. G., 421-422 (Bk. rev.)

Morrison, J. W., 270

Moss, G. I., 373

Mourão, C. A., 127

Mukai, T., 127

Mukherjee, K. L., 104, 239

Mulcahy, D. L., 34, 44

Munsell book of Colora, 404

Müntzing, A., 261, 373

Mutalik, G. S., 145-149, 149

Nakamura, K., 261

Nalimov, O. P., 373

Narbut, S. I., 373

Nash, L. B., 112

Naylor, B., 276

Naylor, J. M., 372

Nei, M., 44

Newberry, K., 307

Newcomer, E. H., 261

Newsom, D. W., 112

Nicolas, D. B., 143

Nicoletti, B., 387

Nichols, L., 158

Nur, U., 261

O'Donald, P., 1-10, 10

Ohta, T., 108, 158

Omenn, G. S. (Ed.), 273-274

Oram, R. N., 332

Oshima, Y., 249

Östergren, G., 261

Oxford, Jennifer, 280

Page, M. M., 284

Pardue, M. L., 121

Parker, R. E., 276

Pateman, J. A., 223, 224

Paul, J., 373

Pavlenko, V. V., 248

Pawsey, S. A., 279

Peacock, W. J., 164

Perkins, J. M., 94, 143, 189-209, 208, 209

Person, C., 224

Peterson, D. G., 85-94, 296

Peterson, J. P., 296

Peterson, P. J., 319

Phillips, M. M., 249

Pickford, R. W., 149

Pierce, W. P., 373

Polani, P. E., 421 (Bk. rev.)

Polito, L., 143

Polyakova, T. F., 373

Pommer, G., 332

Pomper, S., 249

Poole, A. R., 104

Post, R. H., 149 
Poulik, M. D., 127, 401

Powell, J. R., 105-108, 108, 127, 151-158, 158

Power, J. B., 104

Prat, S., 348

Prévost, G., 239

Price, D. J., 211-217, 217

Pryor, A. J., 397-401, 401

Putwain, P. D., 44

Race, R. R., 278

Radford, A., 283

Rai, K. S., 225-230, 230

Rajki, S. (Ed.), 275-276

Ramanis, Z., 270

Rana, M. S., 389-396, 396

Rao, P. N., 412-414

Ravdonikas, L. E., 248

Rees, H., 118-122, 121, 143, 296, 373, 387

Reinbergs, E., 270

Reisfeld, R. A., 104

Reuther, W., 348

Rhoades, M. M., 387

Richmond, R. C., 108, 158

Riede, W., 44

Riley, R., 181, 414

Risueño, M. C., 387

Ritossa, F., 143

Roberts, D. F. (Ed.), 272-273

Robertson, A., 348, 349-356

Rohringer, R., 224

Roman, H., 248, 249

Rosenkrantz, H. S., 373

Ross, M. D., 34, 44, 51, 113-118, 118

Roth, R., 249

Rutishauser, A., 261

Sager, R., 270

Saito, H., 249

Salisbury, E. J., 396

Salzano, F. M., 149

Samborski, D. J., 224

Sandler, L., 386, 387

Sands, S. M., 249

Sannomiya, M., 251-265, 261

Sarkar, P., 181

Sastry, G. R. K., 282

Saunders, J. H., 72

Saura, A., 108

Scandalios, J. G., 401

Scazzocchio, C., 285

Schapp, T., 217

Scheffé, H., 356

Schoefl, G. I., 238

Schreiber, E., 373

Schroeder, H. E., 332

Schwartz, D., 401

Schweizer, D., 122

Seal, H., 209

Selander, R. K., 127

Setterfield, G., 373
Shaw, C. R., 401

Shaw, M., 372

Shaw, R. F., 44, 51, 118

Sheperd, K. W., 269

Sheppard, P. M., 217, 348

Sidhu, G., 219-224, 224

Simchen, G., 217, 223, 224, 387

Simmonds, N. W., 109-112

Simpson, G. G., 409

Singh, R. B., 44

Singh, R. P., 72

Slater, E. T. O., 277

Smith, B. W., 34, 44

Smith, C. A. B., 278

Smith, Derek, 130-131 (Bk. rev.)

Smith, D. A., 412

Smith, I., 401

Smith, R. A. H., 348

Smith, R. L., 332

Snedecor, G. W., 83, 307

Snoed, B., 143

Snow, R., 261

Southern, D. I., 387

Sparkes, C. A., 409-412

Sparrow, D. H., 269

Spassky, B., 108

Spickett, S. G., 356

Stamberg, J., 238, 387

Stebbins, G. L., 181

Stegun, I. A., 356

Stern, C., 421

Storey, W. B., 34

Strickberger, M. W., 307

Stuart, A., 94, 127, 332

Subak-Sharpe, J. H., 279

Subrahmanyam, N. C., 270

Sun, S., 296

Sunderland, E. (Ed.), 272-273

Sybenga, J., 122, 373

Symko, S., 270

Tai-Ying Ho, 113-118

Takahashi, T., 249

Takano, I., 249

Taylor, A. L., 412

Therrien, C. D., 231-239, 239

Thoday, J. M., 356, 406, 406-409

Thompson, K. F., 188

Timmis, J. N., 143, 282

Tinbergen, L., 404

Tinney, F. W., 332

Tracey, M. L., 127

Trippa, G., 387

Trotter, C. D., 412

Tucker, C. L., 332

Turner, R. G., 296, 297, 319, 348

Unrau, I., 181

Upadhyaya, K. C., 104

Urquhart, C., 297, 319 
Valdeyron, G., 34

Van der Plank, J. E., 224

Vardi, Aliza, 171-181, 181

Ventura, S., 231-239

Verma, J. P., 72

Verma, S. C., 118-122

Vosa, C. G., 122

Wallace, A., 348

Wallace, B., 108

Walley, K. A., 309-319

Wally, K., 297

Watkins, J. F., 238

Weimark, A., 188

Weir, B. S., 118

West, D. A., 307

Westergaard, M., 34, 44

Westerman, Jane M., 209

Whaley, W. G., 373

Whelan, E. D. P., 387

White, M. J. D., 261, $422-423$ (Bk. rev.)

Whitehouse, H. L. K., 230, 387

Wigan, L. G., 297

Wilkie, D., 282

Wilkins, D. A., 297

Wilkinson, G. N., 93

Williams, W., 34, 44
Williamson, M., 217

Wills, C. J., 307

Wilson, E. O., 406

Wilson, J. F., 104

Wimber, D. E., 164

Wolters, A. H. G., 122

Woodell, S. R. J., 43

Woods, R. A., 283

Woodward, J., 373

Workman, P. L., 331

Wostenholme G. E. (Ed.), 271-272

Wright, A. J., 188

Wright, E. D., 279

Wright, R. E., 239

Yanagi, T., 373

Yang, S. Y., 127

Yates, F., 94, 208

Yemma, J. J., 231-239, 239

Young, C. S. H., 239

Zabka, G. C., 103, 239

Zarchi Y., 217

Zohary, D., 181

Zuk, J., 44 